\title{
Resurgence of syphilis in England: time for more radical and nationally coordinated approaches
}

After almost two decades of consistent decline, new diagnoses of infectious syphilis in England and Wales are again on the increase. ${ }^{1}$ Recent KC60 reports from genitourinary medicine (GUM) clinics indicate that since 1996, diagnoses of primary and secondary syphilis have more than doubled in males (from 84 to 248) and females ( 32 to 73). The rise was especially marked between 1999 and 2000 , when infections rose by over $160 \%$ (153 to 248 ) in males and $130 \%$ (55 to 73 ) in females, and diagnoses attributed to sex between men rose from 52 to 113 . Since 1997, when the Bristol outbreak heralded the resurgence of syphilis, ${ }^{2}$ subsequent outbreaks have been reported in the North West, South East, and London regions, such that, by 2000, nearly two thirds of nationally reported cases were diagnosed in these areas. Similar outbreaks have also been reported in several large metropolitan $\mathrm{US}^{34}$ and European ${ }^{5}{ }^{6}$ cities. Nearly all have occurred in previously low prevalence areas or among population subgroups in which the disease had been largely eliminated. In addition, the outbreaks were characterised by rapid increases in sexual networks with high rates of partner change; links (travel or migration) with high incidence areas; an increasing predominance of homosexual transmission with a high proportion of HIV co-infection among incident cases.

Why are these outbreaks occurring?

The term outbreak is usually used to describe a greater than anticipated, and often rapid, increase in the levels of an infectious disease in a given time. The outbreak may be followed by subsequent decline in disease incidence either because control measures have taken effect or because of exhaustion of susceptible individuals. In the context of sexually transmitted infections (STIs), there has been some debate as to whether the term should also be used to describe events such as linked cases of disease or a case(s) of congenitally acquired infection, both of which require additional, or reorganisation of, resources for effective management.

Outbreaks of STIs result from an interaction between biological characteristics of the relevant organisms, the behaviours that transmit them, and the effectiveness of prevention and control interventions. The recent outbreaks of syphilis reflect both qualitative and quantitative changes in the spread networks (core groups) which facilitate rapid disease transmission. In their dynamic topology of STI transmission, Wasserheit and $\mathrm{Aral}^{7}$ argue that effective STI prevention programmes, by shortening the duration of infectiousness of an STI, drive up the rate of sex partner change needed to maintain a reproductive rate above 1 . Thus the STI becomes increasingly concentrated within smaller spread networks which encourage STI propagation by virtue of their higher rates of sexual partner change or poor access to curative services. Thus, in the United States, high rates of infection became increasingly concentrated among urban ethnic minority groups in the south eastern states, associated with crack cocaine use. In Britain, widespread behavioural modification in response to HIV/AIDS between the mid-1980s and mid-1990s undoubtedly contributed to dramatic declines in syphilis rates initially among the general population and subsequently among homosexual men (fig 1). ${ }^{8}$ The recent outbreaks of syphilis among HIV infected homosexual men reflect further disease concentration within subgroups with even higher rates of partner change. ${ }^{9}$ These smaller, more concentrated networks may in turn limit the continued effectiveness of syphilis control programmes owing to their lack of accessibility or non-compliance with prevention measures.

Quantitatively, marked changes in the size and distribution of potential spread networks have also occurred since the mid-1990s. Among homosexual men, effective antiretroviral therapies have increased the prevalent pool of HIV infected individuals, many of whom have resumed or increased their sexual activity. Treatment optimism and safer sex fatigue have driven increases in the proportion of homosexual men reporting unsafe sex with anonymous partners. Growth in traditional (for example, saunas, cruising grounds) and new sexual market places (for example, internet chat rooms), within and outside of major urban centres has increased the opportunities for partner acquisition. These sexual networks provide an ideal platform for facilitating rapid STI transmission.

\section{Controlling outbreaks}

The assembled papers in this issue of Sexually Transmitted Infections illustrate some of the difficulties experienced in controlling syphilis outbreaks once established. In all of the affected sites, rapid and accurate diagnosis and treatment of symptomatic patients; effective screening and timely treatment of those at increased risk; and timely treatment of asymptomatic partners have been employed with varying success. Such interventions appear to have been especially important in controlling heterosexual transmission of syphilis in the United States, resulting in plummeting rates of new diagnoses since the mid-1990s. ${ }^{10}$ In contrast, controlling syphilis outbreaks among homosexual men has been considerably more challenging. Enhanced surveillance and research evidence suggest that network members include men with very high rates of partner change, and HIV co-infection, who use venues which facilitate casual partner acquisition (for example, cruising grounds, internet, saunas), and report substantial recreational drug use. ${ }^{9}$ The high rates of partner change and

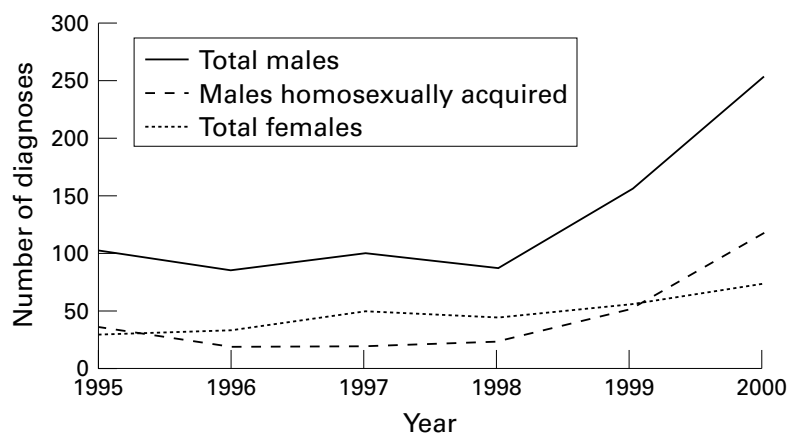

Figure 1 New diagnosis of primary and secondary syphilis, by sex and male acquisition, England and Wales, 1995-2000. 
anonymous partnerships severely limit the utility of partner notification. Unprotected oral sex, while presenting a relatively safer alternative to penetrative sex with regard to HIV transmission, is a very effective way of transmitting syphilis. ${ }^{11}$ Failure to effectively rupture transmission chains within the initial spread networks has probably resulted in extension of the outbreak to adjoining and distant cities (through travel, migration, etc) and to lower risk "maintenance" networks.

Thus, faced with the prospect of growing endemicity, more radical, coordinated approaches to syphilis outbreak control are required. Multidisciplinary working involving collaboration between public health, GUM, microbiology, and infectious diseases colleagues, must be used to develop and implement evidence based interventions that are enhanced to reflect the unique nature of the spread network. Such a strategy has been advocated in the recently produced guidelines for managing STI outbreaks ${ }^{12}$ developed by CDSC in partnership with the Association for Genitourinary Medicine (AGUM), the Medical Society for the Study of Venereal Diseases (MSSVD), and the Public Health Medicine Environmental Group (PHMEG) (currently out for wider consultation on the PHLS website: www.phls.co.uk). Those involved in disease control must work more closely with laboratory experts to facilitate improved diagnosis and screening of at-risk individuals. In particular, recent outbreaks have highlighted the urgent need for the development and evaluation of rapid, sensitive, and specific syphilis diagnostic tests, especially salivary assays, for use in the field. Enhanced local STI surveillance systems are also needed to ensure rapid and timely reporting of incident cases; to collect demographically and behaviourally relevant data to inform public heath action; and to evaluate the impact of prevention interventions.

We must continue to develop innovative interventions which are appropriate, accessible, and acceptable to those at greatest risk and who are currently driving disease transmission. Routine and opportunistic syphilis screening, with easy and rapid treatment of all those found positive (for example, fast track, open access syphilis screening slots in clinics), remains key. HIV positive homosexual men attending HIV and GUM clinics need this as well as proactive syphilis education. Syphilis screening needs to be extended to venues where transmission may be occurring (for example, saunas and cruising grounds). Clearly, oral (saliva) tests will be critical to achieving this, and must be developed speedily. Other vulnerable networks (internet chat rooms) need to be identified and targeted with a view to limiting disease transmission and rupturing transmission chains with other spread networks (for example, by utilising social and geographic network information to direct targeted health promotion and screening interventions). Mass screening and treatment interventions have been attempted elsewhere and may now need to be considered in worst affected areas. Simple treatment, preferably with a single dose regimen, is also needed. The most user friendly test will not catch on if it can result in 2 weeks of daily injections.
Finally, evaluation, evaluation, evaluation! Interventions should be evaluated to determine their effectiveness; to rationally inform the withdrawal, continuation, or redeployment of available resources; and to contribute to the relatively sparse evidence base on effective interventions for controlling STI outbreaks. In this regard, a strong case could be made for adopting a national coordinated approach for reviewing and evaluating syphilis outbreak control. Given its mandate, such national coordination by $\mathrm{CDSC}^{13}$ (or SCIEH in Scotland), although not mentioned in the Sexual Health Strategy, would greatly facilitate pooling of resources, sharing of expertise, and learning across epicentres. Gains of the past two decades should not be so easily surrendered and a foe as formidable as syphilis certainly deserves a formidable response.

HIVISTI Division, PHLS Communicable Disease Surveillance Centre, 61 Colindale Avenue, London NW9 5EQ and Department of Sexually Transmitted Diseases, Royal Free and University College Medical School, Mortimer Market Centre, off Capper Street, London WC1E 6AU, UK

ANGUS NICOLL

HIVISTI Division, PHLS Communicable Disease Surveillance Centre, 61 Colindale Avenue, London NW9 5EQ, UK

GEORGE KINGHORN

Royal Hallamshire Hospital, Gossop Road, Sheffield S10 2fF, UK

Correspondence to: Dr Kevin A Fenton, HIV/STI Division, PHLS

Communicable Disease Surveillance Centre, 61 Colindale Avenue, London NW9 5EQ, UK

kfenton@phls.org.uk

1 CDSC. Diagnoses of gonorrhoea reach ten-year high. Commun Dis Rep CDR Wkly [serial online] 2001;11 (15). Available at www.phls.co.uk/ publications/CDR\%20Weekly/archive/news 3001 html\#gonorrhoea

2 CDSC. Syphilis in Bristol 1997-8: an update. Commun Dis Rep CDR Wkly 1998;8:413

3 CDC. Outbreaks of syphilis among men who have sex with men-southern California, 2000. MMWR Morb Mort Wkly Rep 2001;50:117-20.

4 CDC. Resurgent bacterial sexually transmitted disease among men who have sex with men-King County, Washington, 1997-1999. MMWR Morb Mort Wkly Rep 1999;48:773-7.

5 Dupin N, Jdid R, N'Guyen YT, et al. Syphilis and gonorrhoea in Paris: the return. AIDS 2000;15:814-15.

6 Hopkins S, Courtney G, O'Connell T, et al. Re-emergence of syphilis in Dublin. Oral presentation at the MSSVD Spring Meeting in Belfast, 27-29 May 2001.

7 Wasserheit JN, Aral SO. The dynamic topology of sexually transmitted disease epidemics: implications for prevention strategies. 7 Infect Dis 1996;174(Suppl 2):S201-13.

8 Nicoll A, Hughes G, Donnelly M, L et al. Assessing the impact of national anti-HIV sexual health campaigns: trends in the transmission of HIV and other sexually transmitted infections in England. Sex Transm Inf 2001;77:242-7.

9 Clark P, Cook P, Syed Q, et al. Re-emerging syphilis in the North West: lessons from the Manchester outbreak. Liverpool: John Moores Unversity, 2001.

10 Centres for Disease Control and Prevention. The national plan to eliminate syphilis from the United States. Division of STD Prevention, Centres for Disease Control and Prevention. October, 1999

11 Flemming DT, Wasserheit JN. From epidemiological synergy to public health policy and practice: the contribution of other sexually transmitted diseases to sexual transmission of HIV infection. Sex Transm Inf 1999;75:317.

12 CDSC. Guidelines for managing local outbreaks of sexually transmitted infections. An outbreak plan. Produced in association with AGUM, MSSVD and PHMEG, 2001

13 Department of Health and Social Security. Health Service Development. Co-ordination of epidemiological services for communicable diseases and food poisoning: Communicable Disease Surveillance Centre. Health Circular HC(80)2, LAC (80)1 DHSS, London: February, 1980. 